\title{
Emergent reduced dimensionality by vertex frustration in artificial spin ice
}

\author{
Ian Gilbert ${ }^{1}$, Yuyang Lao ${ }^{1}$, Isaac Carrasquillo', Liam O'Brien ${ }^{2,3}$, Justin D. Watts ${ }^{2,4}$, Michael Manno², \\ Chris Leighton ${ }^{2}$, Andreas Scholl ${ }^{5}$, Cristiano Nisoli ${ }^{6}$ and Peter Schiffer ${ }^{1 \star}$
}

\begin{abstract}
Reducing the dimensionality of a physical system can have a profound effect on its properties, as in the ordering of low-dimensional magnetic materials ${ }^{1}$, phonon dispersion in mercury chain salts ${ }^{2}$, sliding phases $^{3}$, and the electronic states of graphene ${ }^{4}$. Here we explore the emergence of quasi-one-dimensional behaviour in two-dimensional artificial spin ice, a class of lithographically fabricated nanomagnet arrays used to study geometrical frustration ${ }^{5-7}$. We extend the implementation of artificial spin ice by fabricating a new array geometry, the so-called tetris lattice ${ }^{8}$. We demonstrate that the ground state of the tetris lattice consists of alternating ordered and disordered bands of nanomagnetic moments. The disordered bands can be mapped onto an emergent thermal one-dimensional Ising model. Furthermore, we show that the level of degeneracy associated with these bands dictates the susceptibility of island moments to thermally induced reversals, thus establishing that vertex frustration can reduce the relevant dimensionality of physical behaviour in a magnetic system.
\end{abstract}

Arrays of interacting, single-domain nanomagnets known as artificial spin ice have proved to be useful model systems with which to experimentally explore the microscopic nature of geometrical frustration $^{6-8}$. Simple square and kagome artificial spin ice lattices have been subject to intense study because they provide unique insight into the consequences of frustrated magnetism, such as residual entropy $y^{9,10}$ and magnetic charge excitations ${ }^{11-14}$. In the past few years, improved thermalization methods have provided access to the low-energy phases of artificial spin ice structures ${ }^{10,15,16}$ and even their dynamics ${ }^{17-19}$. There is now growing awareness that one could even employ these artificial systems to design desired emergent behaviours and exotic states ${ }^{20}$. To this end, a number of new lattice geometries have been proposed to produce novel emergent phenomena through 'vertex frustration', where the lattice geometry forces groups of neighbouring island moments, called vertices, into higher-energy configurations that would not otherwise be observed in the ground state ${ }^{8,21}$. Here we combine these theoretical and experimental advances to study thermal fluctuations about the ground state of the tetris lattice ${ }^{8}$, a regular two-dimensional lattice with reduced symmetry that is designed to exhibit vertex frustration. By analysis of the thermalized ground state, we demonstrate that the lattice decomposes into alternating stripes of two kinds, one of which exhibits the expected disordered state for a ferromagnetic one-dimensional Ising model.

The structure of the tetris lattice is depicted in Fig. 1; it is a decimation of the square lattice in which $3 / 8$ of the islands
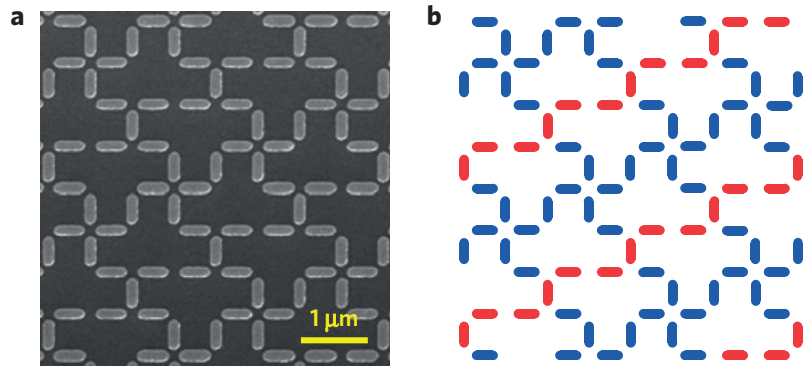

Figure 1 | The structure of the tetris lattice. The tetris lattice is a complicated decimation of the artificial square spin ice lattice. $\mathbf{a}$, Scanning electron micrograph of a tetris lattice with $600 \mathrm{~nm}$ island separation. $\mathbf{b}$, The lattice is most easily understood by decomposition into two types of diagonal stripes. The backbones (blue) are comprised of chains of four- and two-vertex islands and, in the ground state, are completely ordered. The staircases (red) are comprised of pairs of horizontal islands that map onto emergent one-dimensional Ising spins, joined by single vertical islands between them.

are removed. Its low-energy behaviour is best understood by decomposing the lattice into parallel strips of 'backbones' (blue in Fig. 1b) containing four-island vertices and 'staircases' (red in Fig. 1b) containing three-island vertices. This lattice is vertexfrustrated because it is impossible to place each three-island vertex of the staircases into its minimum-energy Type A configuration (the types of vertices found in the tetris lattice are enumerated in Fig. 2a). The vertex frustration breaks the ground state order of the parent square lattice, forcing half of the three-island vertices out of their Type A ground state into the higher-energy Type B configuration.

A simple vertex-model theory ${ }^{22}$ for this geometry predicts ordered backbones and disordered staircases ${ }^{8}$. Although in principle the staircases do have a completely ordered state (Fig. 2b), in practice the many disordered configurations (that is, the ice manifold, one state of which is depicted in Fig. 2c) cause order on the staircases to be entropically suppressed. Indirect interactions ${ }^{23}$ may also play a role in preventing the alignment between backbones necessary for the ordered state to develop. In the ice manifold (which is analogous to the kagome ice I phase of artificial kagome spin ice ${ }^{24}$ ), each step of the staircases must contain exactly one Type B vertex. These Type B vertices can be placed along the staircase with a freedom that leads to a finite entropy density. The horizontal island moments of each step, comprising two parallel islands, can be chosen at will in the ice manifold at low temperature (Fig. 2c), defining

\footnotetext{
${ }^{1}$ Department of Physics and Frederick Seitz Materials Research Laboratory, University of Illinois at Urbana-Champaign, Urbana, Illinois 61801, USA ${ }^{2}$ Department of Chemical Engineering and Materials Science, University of Minnesota, Minneapolis, Minnesota 55455, USA. ${ }^{3}$ Thin Film Magnetism Group, Department of Physics, Cavendish Laboratory, University of Cambridge, Cambridge CB3OHE, UK. ${ }^{4}$ School of Physics and Astronomy, University of Minnesota, Minneapolis, Minnesota 55455, USA. ${ }^{5}$ Lawrence Berkeley National Laboratory (LBNL), Berkeley, California 94720, USA. ${ }^{6}$ Theoretical Division and Center for Nonlinear Studies, MS B258, Los Alamos National Laboratory, Los Alamos, New Mexico 87545, USA. *e-mail: pschiffe@illinois.edu
} 
a

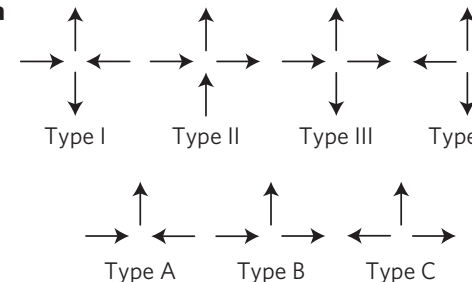

b

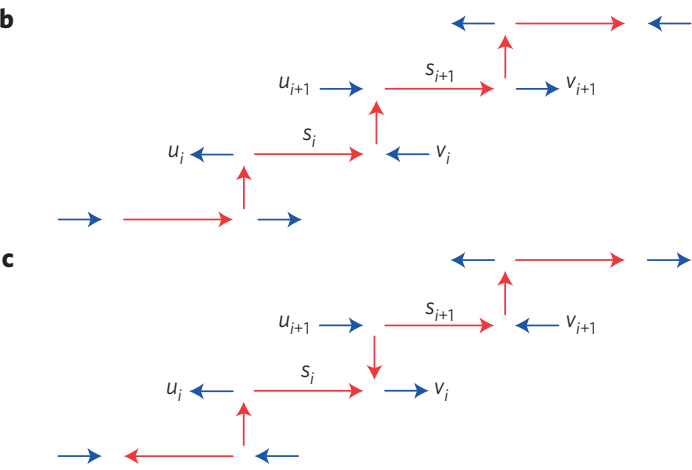

Figure 2 | Vertices and staircases in the tetris lattice. a, A catalogue of the types of vertices that can occur in the tetris lattice. For each class (four-island, three-island, and so on), the types are listed in order of increasing energy. b, A ferromagnetically ordered staircase in which all the island moments are arranged head-to-tail. c, A disordered staircase. The large number of disordered configurations arises from the freedom in allocating Type $A$ and $B$ vertices. The labels in $\mathbf{b}$ and $\mathbf{c}$ define the correlations shown in Fig. $4 \mathrm{~b}$. The long arrows, $s_{i}$, correspond to Type a vertices, that is, two regular islands magnetized parallel to one another.

an emergent one-dimensional Ising system which, in the vertexmodel approximation, exhibits an effective ferromagnetic coupling between neighbouring steps (see the Supplementary Information for a detailed derivation). Thus at zero temperature, the vertex-model degeneracy of the two-dimensional tetris lattice can be subsumed into the statistical mechanics of a one-dimensional Ising system in a fictitious thermal state.

We experimentally investigated the tetris lattice's ice manifold predicted by the vertex model by fabricating tetris artificial spin ice lattices with single-domain permalloy $\left(\mathrm{Ni}_{81} \mathrm{Fe}_{19}\right)$ islands (nominal island dimensions of $470 \times 170 \times 3 \mathrm{~nm}$ and island spacings of $a=600 \mathrm{~nm}$ and $800 \mathrm{~nm}$ ). The energy barrier to reversal of the magnetic moment of these islands is approximately equal to the thermal energy available at room temperature ${ }^{17,18}$ (owing to the low thicknesses of $3-3.5 \mathrm{~nm}$ ), so thermal fluctuations cause the island moments to flip rapidly at room temperature. The exact mechanism of island moment reversal (via coherent rotation or more complex intermediate state ${ }^{25}$ ) is unclear, but by slowly cooling below room temperature we can drive the arrays into their thermalized ground state. This sort of sample allows us to study both the ground state and thermal fluctuations about the ground state using photoemission electron microscopy with X-ray magnetic circular dichroism contrast (XMCD-PEEM; refs 26,27).

Measurements of the island moment configurations at $T=160 \mathrm{~K}$ indicate that the arrays are static at that temperature and are close to their predicted ground state, as depicted in Fig. 3. The backbone islands exhibit the predicted long-range order, as can be seen from the alternating chains of five white and five black islands in Fig. 3a, or from the ordered blue arrows in Fig. 3b. The staircases, however, do not seem to be ordered. We characterize the system by considering the population of the different vertices in the lattice. As shown in Fig. $4 a$, the vertex populations (defined in Fig. 2a) extracted from the images are close to those expected for the ground state. For
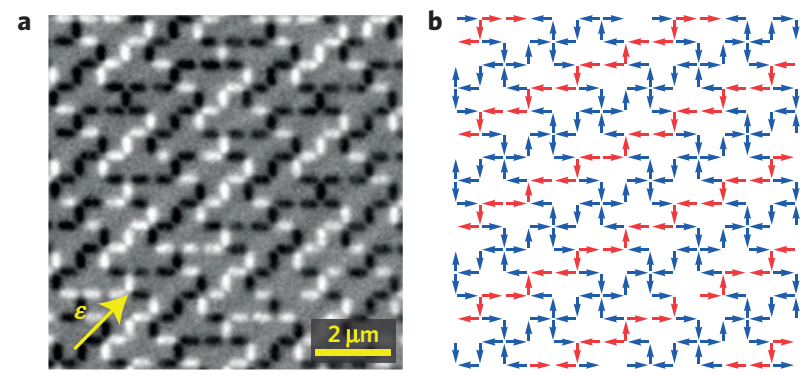

Figure 3 | The experimental ground state of the tetris lattice.

a, XMCD-PEEM image of a $600 \mathrm{~nm}$ tetris lattice. The black/white contrast indicates whether the magnetization of an island has a component parallel or antiparallel to the polarization of the incident X-rays, which is indicated by the yellow arrow. b, Map of the moment configurations in a. As in Fig. 1, island moments belonging to the backbones are coloured blue, and moments belonging to the staircases are red. The backbone island moments are ordered, which can be seen by examining the arrows in $\mathbf{b}$ or noting the diagonal segments of five islands with alternating black/white contrast in a. The staircase moments, however, are not ordered.

example, nearly all of the four-island vertices observed are in the lowest-energy Type I configuration.

The presence or absence of order on the staircases is related to the relative alignment of the moments in the neighbouring backbones $^{8}$. In particular, disordered staircases require antiparallel neighbouring backbone moments for a given step (for example, $u_{i}$ and $v_{i}$ in Fig. 2). Therefore, as a first step towards characterizing the state of the staircases, we consider several correlations between the backbone islands, $\left\langle u_{i} u_{i+1}\right\rangle,\left\langle u_{i+1} v_{i}\right\rangle$ and $\left\langle u_{i} v_{i}\right\rangle$. If the extensively degenerate disordered staircase configurations indeed dominate, these correlations must be approximately $-1,1$ and -1 , respectively. Note that although this antiparallel configuration of neighbouring backbone moments is also consistent with two ordered states in which all the staircase islands are aligned head-to-tail, these ordered configurations would yield $\left\langle s_{i} s_{i+1}\right\rangle=1$. From Fig. $4 \mathrm{~b}$, we see that the correlations $\left\langle u_{i} u_{i+1}\right\rangle,\left\langle u_{i+1} v_{i}\right\rangle$ and $\left\langle u_{i} v_{i}\right\rangle$ extracted from the experimental data indeed match these predictions closely and $\left\langle s_{i} s_{i+1}\right\rangle \neq 1$, indicating that the staircases are indeed disordered and that the ordered staircase configuration of Fig. $2 b$ is entropically suppressed. Furthermore, the measured correlations between farther-neighbour staircase islands (Fig. 4c) are consistent with the exact result for the one-dimensional Ising model, indicating that the one-dimensional Ising model serves as a good approximation for the staircase island moments and underscoring the one-dimensional nature of the staircases.

The nearest-neighbour correlation $\left\langle s_{i} s_{i+1}\right\rangle$ between staircases' horizontal islands is higher than that predicted by the vertex model alone, which ignores the long range of the dipolar interaction between the steps. Although disorder in the islands' magnetic properties can affect the correlations present in thermalized artificial spin ice $^{28}$, we attribute the bulk of this discrepancy to long-range dipolar interactions, which can be included by introducing next-nearestneighbour interactions in the form of charge-charge terms $s^{10,24}$. A calculation presented in the Supplementary Information shows how this addition simply renormalizes the coupling of the nearestneighbour Ising system. Using micromagnetic estimates for the charge-charge interactions, we obtain $\left\langle s_{i} s_{i+1}\right\rangle=0.66$, close to the measured value of 0.60 .

Not only does the large manifold of disordered staircase configurations suppress order in the staircases, but its energy landscape also plays a crucial role in determining the dynamics. To explore the effect of geometry on thermal fluctuations, we took series of images of an $a=800 \mathrm{~nm}$ tetris lattice as the temperature was varied from $280-360 \mathrm{~K}$. These images were compiled into 

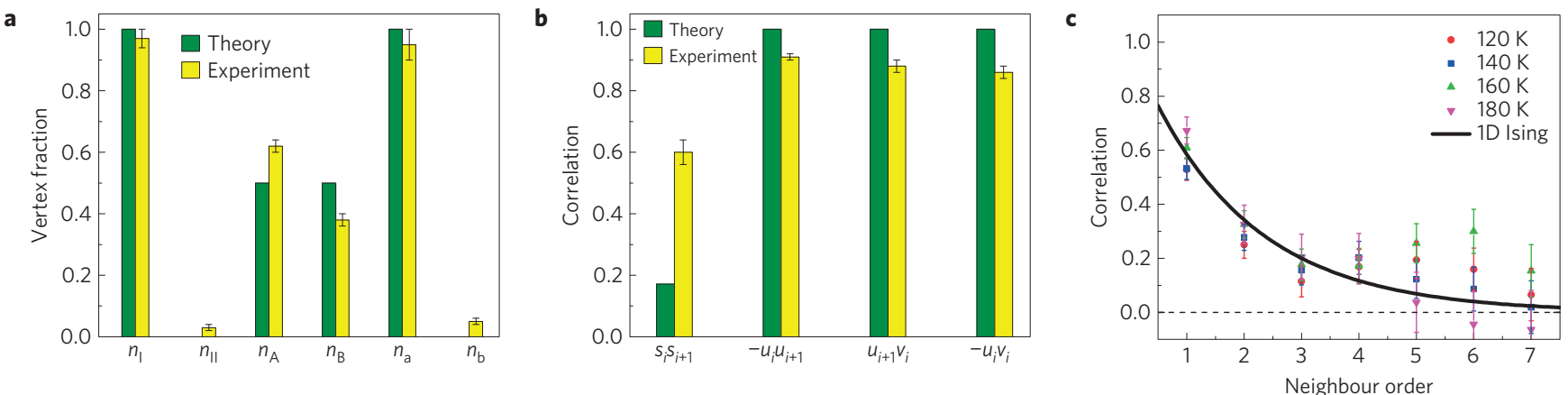

Figure 4 | Vertex populations and moment correlations. a, Vertex populations predicted by a simple vertex-model theory (green) and measured experimentally on $\sim 10,000$ islands in a $600 \mathrm{~nm}$ tetris lattice with $3 \mathrm{~nm}$ thick islands at $160 \mathrm{~K}$ (yellow). None of the energetically costly Type III, IV, or C vertices were observed in any of the images. Error bars correspond to the square root of the number of vertices of each type. $\mathbf{b}$, Correlations predicted by theory (green) and measured experimentally (yellow). The correlations among the backbone moments compare favourably with theory. The correlation $\left\langle s_{i} s_{i+1}\right\rangle$ between the emergent Ising spins of the staircases differs markedly from the simple vertex theory (but can be explained by longer-range dipolar interactions, as detailed in the Supplementary Information). c, Correlation between the staircase spins $s_{i}$ as a function of neighbour order. The black curve is the exact result for the 1D Ising model with the interaction energy chosen to fit the experimental data (see Supplementary Information for details). The coloured points are experimental data taken at various temperatures (moment freezing in this sample occurred below $T \sim 200 \mathrm{~K}$, and the moments were

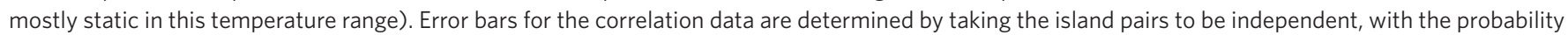
for favourable alignment of a given pair determined by a binomial distribution.
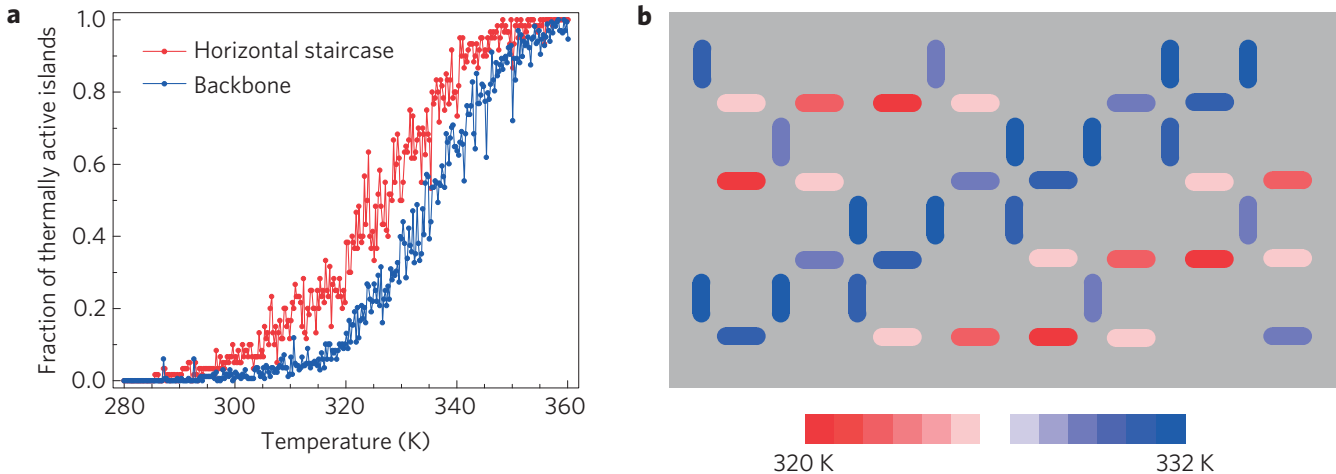

Figure $\mathbf{5}$ | Thermally active islands as a function of temperature. $\mathbf{a}$, The fraction of backbone islands (blue) and horizontal staircase islands, that is, the $s_{i}$, (red) that are thermally active as a function of temperature. The data are extracted from the movie taken on heating given in the Supplementary Information. Here an island is defined as thermally active if it flips at a sufficiently high rate (approximately every five seconds) that its XMCD contrast is lost during the $12 \mathrm{~s}$ exposure. The offset between the curves indicates that the staircase islands become thermally active about ten degrees below the point at which the backbone islands begin to fluctuate. Approximately 360 islands per frame were analysed. $\mathbf{b}, \mathrm{A}$ unit cell of the tetris lattice. Each island is shaded according to the temperature at which half of the islands of that type lose XMCD contrast in the heating movie. Consistent with panel $\mathbf{a}$, the staircase islands $s_{i}$ reach this level of thermal fluctuations about $10 \mathrm{~K}$ lower than the backbone islands.

movies demonstrating the dynamics, in which one can observe the relationship between fluctuation rates and temperature (see Supplementary Information and Supplementary Movies). At low temperatures, in proximity to the ground state, the thermally active island moments (visible as islands whose contrast reverses from black to white, or vice versa, between subsequent images) are confined to the vertex-frustrated staircases. At high temperatures, however, all the island moments in the array fluctuate so rapidly that the XMCD contrast is washed out to a uniform grey. Figure 5a and $\mathrm{b}$, which show the fraction of backbone and staircase islands that are thermally active as a function of temperature and the temperatures at which the different types of islands in the tetris lattice unit cell become thermally active, respectively, both confirm that the onset of thermal fluctuations lies at a significantly lower temperature for the staircase moments.

Whereas the ordered backbones have just two degenerate ground state configurations separated by a large energy barrier, the disordered staircases can explore many different disordered configurations through single island moment reversals that rearrange the Type A and B vertices. This again is consistent with a one-dimensional Ising model. Interestingly, when the temperature is high enough for the backbone island moments to fluctuate, they reverse in a correlated fashion with entire five-island segments reversing together, reminiscent of natural frustrated magnetic materials in which the fluctuating degrees of freedom are not individual spins, but clusters of spins ${ }^{29}$.

In summary, we have found that the lower symmetry of the tetris lattice compared to the original artificial square spin ice (twofold without mirror symmetry versus four-fold with mirror symmetry) allows the lattice to decompose into alternating quasi-onedimensional bands: the backbones and the staircases. The backbones are not vertex-frustrated and consequently take on an ordered configuration that is comparatively robust against fluctuations. Vertex frustration in the staircase bands, however, leads to disorder, which can be described by an emergent one-dimensional Ising model, and to susceptibility to thermal fluctuations. This system therefore demonstrates how vertex frustration, rather than geometrical separation or a fortuitous distribution of exchange interactions, can lead to an effectively reduced dimensionality in a many-body magnetic system. 
Within the ice manifold of the tetris lattice, much remains to be explored. At lower entropies, the dipolar interactions between the disordered spins (both within and between staircases), as well as with defects within the ordered backbones, should provide a complex energy profile where competition might lead to different stages of ordering, mixed transitions, domain formation, and a potentially rich phase diagram. More generally, natural magnetic materials with such interesting emergent reduced dimensionality are typically found serendipitously, and it is necessarily difficult to tune the strength of the various interactions. Artificial spin ice, however, has matured from a model of geometrical frustration towards a testbed where lattice geometry can be intentionally exploited to forge complex states in the low-energy behaviour of a magnetic system. In addition to facilitating investigations of fundamental physics phenomena, the ability to tune the onset of thermal fluctuations in a magnetic material through its geometry may prove useful in future magnetic recording technology $\mathrm{y}^{30}$.

\section{Methods}

Methods and any associated references are available in the online version of the paper.

\section{Received 10 April 2015; accepted 16 September 2015;} published online 26 October 2015

\section{References}

1. de Jongh, L. J. \& Miedema, A. R. Experiments on simple magnetic model systems. Adv. Phys. 23, 1-260 (1974).

2. Hastings, J. M. et al. One dimensional phonons and "phase-ordering" transition in $\mathrm{Hg}_{3-\delta} \mathrm{AsF}_{6}$. Phys. Rev. Lett. 39, 1484-1487 (1977).

3. O'Hern, C. S., Lubensky, T. C. \& Toner, J. Sliding phases in XY models, crystals, and cationic lipid-DNA complexes. Phys. Rev. Lett. 83, 2745-2748 (1999).

4. Castro Neto, A. H., Guinea, F., Peres, N. M. R., Novoselov, K. S. \& Geim, A. K. The electronic properties of graphene. Rev. Mod. Phys. 81, 109-162 (2009).

5. Wang, R. F. et al. Artificial 'spin ice' in a geometrically frustrated lattice of nanoscale ferromagnetic islands. Nature 439, 303-306 (2006).

6. Heyderman, L. J. \& Stamps, R. L. Artificial ferroic systems: Novel functionality from structure, interactions and dynamics. J. Phys. Condens. Matter 25, 363201 (2013).

7. Nisoli, C., Moessner, R. \& Schiffer, P. Colloquium: Artificial spin ice: Designing and imaging magnetic frustration. Rev. Mod. Phys. 85, 1473-1490 (2013).

8. Morrison, M. J., Nelson, T. R. \& Nisoli, C. Unhappy vertices in artificial spin ice: New degeneracies from vertex frustration. New J. Phys. 15, 045009 (2013).

9. Lammert, P. E. et al. Direct entropy determination and application to artificial spin ice. Nature Phys. 6, 786-789 (2010).

10. Zhang, S. et al. Crystallites of magnetic charges in artificial spin ice. Nature 500, 553-557 (2013).

11. Mengotti, E. et al. Real-space observation of emergent magnetic monopoles and associated Dirac strings in artificial kagome spin ice. Nature Phys. 7, 68-74 (2011)

12. Ladak, S., Read, D. E., Perkens, G. K., Cohen, L. F. \& Branford, W. R. Direct observation of magnetic monopole defects in an artificial spin-ice system. Nature Phys. 6, 359-363 (2010).

13. Phatak, C., Petford-Long, A. K., Heinonen, O., Tanase, M. \& De Graef, M. Nanoscale structure of the magnetic induction at monopole defects in artificial spin-ice lattices. Phys. Rev. B 83, 174431 (2011).

14. Pollard, S. D., Volkov, V. \& Zhu, Y. Propagation of magnetic charge monopoles and Dirac flux strings in an artificial spin-ice lattice. Phys. Rev. B 85, 180402 (2012).
15. Morgan, J. P., Stein, A., Langridge, S. \& Marrows, C. H. Thermal ground-state ordering and elementary excitations in artificial magnetic square ice. Nature Phys. 7, 75-79 (2011).

16. Porro, J. M., Bedoya-Pinto, A., Berger, A. \& Vavassori, P. Exploring thermally induced states in square artificial spin-ice arrays. New J. Phys. 15, 055012 (2013).

17. Farhan, A. et al. Exploring hyper-cubic energy landscapes in thermally active finite artificial spin-ice systems. Nature Phys. 9, 375-382 (2013).

18. Farhan, A. et al. Direct observation of thermal relaxation in artificial spin ice. Phys. Rev. Lett. 111, 057204 (2013).

19. Kapaklis, V. et al. Thermal fluctuations in artificial spin ice. Nature Nanotech. 9, 514-519 (2014).

20. Stamps, R. L. Artificial spin ice: The unhappy wanderer. Nature Phys. 10, 623-624 (2014).

21. Gilbert, I. et al. Emergent ice rule and magnetic charge screening from vertex frustration in artificial spin ice. Nature Phys. 10, 670-675 (2014).

22. Baxter, R. J. Exactly Solved Models in Statistical Mechanics (Academic, 1982).

23. Zhang, S. et al. Ignoring your neighbors: Moment correlations dominated by indirect or distant interactions in an ordered nanomagnet array. Phys. Rev. Lett. 107, 117204 (2011).

24. Möller, G. \& Moessner, R. Magnetic multipole analysis of kagome and artificial spin-ice dipolar arrays. Phys. Rev. B 80, 140409 (2009).

25. Donolato, M. et al. Characterization of domain wall-based traps for magnetic beads separation. J. Appl. Phys. 111, 07B336 (2012).

26. Ade, H. \& Stoll, H. Near-edge X-ray absorption fine-structure microscopy of organic and magnetic materials. Nature Mater. 8, 281-290 (2009).

27. Cheng, X. M. \& Keavney, D. J. Studies of nanomagnetism using synchrotron-based X-ray photoemission electron microscopy (X-PEEM). Rep. Prog. Phys. 75, 026501 (2012).

28. Drisko, J., Daunheimer, S. \& Cumings, J. FePd 3 as a material for studying thermally active artificial spin ice systems. Phys. Rev. B 91, 224406 (2015).

29. Lee, S.-H. et al. Emergent excitations in a geometrically frustrated magnet. Nature 418, 856-858 (2002).

30. Stipe, B. C. et al. Magnetic recording at $1.5 \mathrm{Pbm}^{-2}$ using an integrated plasmonic antenna. Nature Photon. 4, 484-488 (2010).

\section{Acknowledgements}

This work was funded by the US Department of Energy, Office of Basic Energy Sciences, Materials Science and Engineering Division under grant no. DE-SC0010778. The work of C.N. was carried out under the auspices of the US Department of Energy at LANL under contract no. DE-AC52-06NA253962. Work performed at the University of Minnesota (UMN) was supported by the National Science Foundation through the UMN MRSEC under award number DMR-1420013, as well as by EU Marie Curie IOF project no. 299376. The Advanced Light Source is supported by the Director, Office of Science, Office of Basic Energy Sciences, of the US Department of Energy under contract no. DE-AC02-05CH11231

\section{Author contributions}

I.G., C.N. and P.S. designed this study. I.G. and Y.L. fabricated the lithographic patterns, and L.O'B., J.D.W., M.M. and C.L. optimized and deposited the wedged permalloy films A.S., I.G. and Y.L. conducted the XMCD-PEEM measurements. I.C. assisted with data analysis. C.N. developed the theory describing the tetris lattice. I.G., C.N. and P.S. wrote the paper with input from all of the co-authors.

\section{Additional information}

Supplementary information is available in the online version of the paper. Reprints and permissions information is available online at www.nature.com/reprints. Correspondence and requests for materials should be addressed to P.S.

\section{Competing financial interests}

The authors declare no competing financial interests. 


\section{Methods}

Arrays of tetris artificial spin ice with lattice constants of $a=600$ and $800 \mathrm{~nm}$ were patterned on a silicon substrate by means of electron-beam lithography, using a bilayer resist stack with layers of polymethyl methacrylate (PMMA) of two different molecular weights. A permalloy $\left(\mathrm{Ni}_{81} \mathrm{Fe}_{19}\right)$ film was then electron-beam evaporated in ultrahigh vacuum (see prior work ${ }^{5,10}$ for more details) while progressively stepping a growth shutter in front of the sample to produce arrays of varying thicknesses (that is, 'discretized wedges'). Because the rate at which therma fluctuations occur depends very sensitively on island thickness, this ensured that some of the arrays would undergo observable thermal fluctuations near room temperature. We found that 3 to $3.5 \mathrm{~nm}$ thick arrays were thermally active around room temperature, consistent with previous studies ${ }^{17,18}$. A $2 \mathrm{~nm}$ capping layer of $\mathrm{Al}$ was deposited on top of the permalloy to inhibit oxidation. The island dimensions $(470 \times 170 \times 3 \mathrm{~nm})$ were similar to those used in previous studies ${ }^{17,18}$ of thermally active artificial spin ice, which facilitates comparison. Also, because these islands are thermally active around room temperature, problems associated with thicker islands (high temperature annealing and associated complications involving substrate-island interdiffusion ${ }^{10}$ ) are avoided.

The arrays were measured using synchrotron-based photoemission electron microscopy with X-ray magnetic circular dichroism contrast ${ }^{25,26}$ (XMCD-PEEM) at beamline 11.0.1 at the Advanced Light Source. Imaging was performed at the Fe $L_{3}$ edge at $707 \mathrm{eV}$. Most images were an average of nine two-second exposures taken at each helicity, but the individual frames in the Supplementary Movies have a total exposure of only $12 \mathrm{~s}$. Uniform black/white contrast on the islands (which indicates whether the island magnetization has a positive or negative projection on the polarization of the incident X-rays) confirms the islands are single-domain and are effectively giant Ising spins. The images allow us to unambiguously identify the exact microstate of the array, as shown in Fig. 3. 